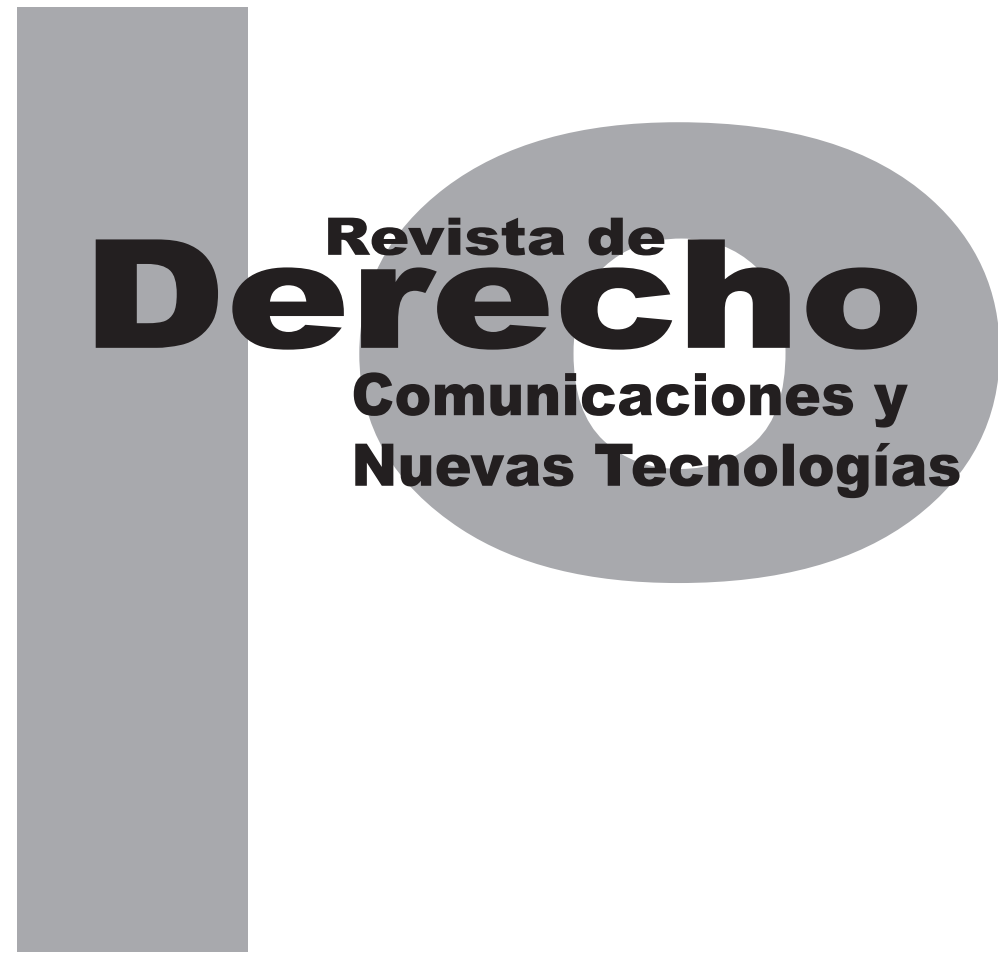

\title{
EL ESPACIO ULTRATERRESTRE: UNA VEZ EL ORIGEN, HOY EL DESTINO
}

\author{
Manuel Guillermo Contreras Henao
}

Universidad de los Andes

Facultad de Derecho

Revista de Derecho, Comunicaciones y Nuevas Tecnologías

No.11, Enero - Junio de 2014. ISSN 1909-7786 


\title{
El espacio ultraterrestre: una vez el origen, hoy el destino ${ }^{1}$
}

\author{
Manuel Guillermo Contreras Henao²
}

\section{RESUMEN}

Conocer la historia de la disciplina que se estudia es la responsabilidad primaria del abogado que se adentra en un nuevo campo. Así, el desarrollo de la humanidad ha sido marcado por la observación de los astros y por la dependencia del hombre del espacio ultraterrestre. En diferentes culturas, el hombre ha basado sus creencias en la observación de las estrellas, y ha buscado ascender al cielo para encontrar a sus creadores. Con el desarrollo tecnológico, el ser humano ha logrado comprender muchos de los misterios que inundan el espacio ultraterrestre. Inclusive, ha llegado a explorar los rincones más recónditos de nuestra galaxia, y, como consecuencia, han surgido nuevas incógnitas. Esta ambición por el conocimiento ha llevado a que el hombre diseñe un sistema jurídico para regu-
Knowing the history of the field subject to study is the primary task of the lawyer that adventures into a new field. As such the development of humanity has been marked by the observation of the stars and the deep dependence on outer space. Different cultures have based their believes on the observation of the stars, and have strived to ascend into the heavens to meet their makers. With technological development, man has managed to comprehend many of the mysteries that rest in outer space. In fact, man has been able to explore the darkest corners of our galaxy and as consequence new queries have emerged. This ambition for knowledge has lead humanity to design a legal system to regulate activities in outer space, and as such achieve a better development of space exploration.

Cómo citar este artículo: Contreras Henao, M. G. (Junio, 2014). El espacio ultraterrestre: una vez el origen, hoy el destino. Revista de Derecho, Comunicaciones y Nuevas Tecnología, 11.

2 Estudiante de décimo semestre de Derecho, con opción en Relaciones Internacionales de la Universidad de los Andes. Correo electrónico: mg.contreras279@uniandes.edu.co 
lar las actividades en el espacio, y para lograr un mejor desarrollo de la exploración espacial.

Palabras Clave: Espacio ultraterrestre, regulación del espacio, estrellas, historia, exploración.
KEYWORDS: Outer space, space regulation, stars, history, exploration. 


\section{SUMARIO}

Introducción - I. LA HISTORIA DEL ESPACIO - II. LA CIENCIA DEL ESPACIO - III. LA EXPLORACIÓN DEL ESPACIO - IV. LA REGULACIÓN DEL ESPACIO - V. CONCLUSIONES - Referencias 


\section{Introducción}

Mirar hacia las estrellas y tratar de comprender el universo ha sido una de las grandes obsesiones del ser humano desde el principio de los tiempos. Cada cultura ha desarrollado su propia explicación del origen del ser humano, y un gran número de estas encuentran sus orígenes en seres celestiales que habitan junto a las estrellas. La tradición cristiana da gran importancia a la observación de los astros, pues la observación de estos, según el Nuevo Testamento, permite a los Reyes Magos llegar hasta el lugar de nacimiento del Niño Jesús. Miles de años antes de este suceso, los antiguos egipcios lograron desarrollar la primera carta astral, lo que se vio reflejado en la construcción de las pirámides de Guiza, las cuales siguen el patrón de la constelación del Cinturón de Orión (Von Spaeth).

Como se expresó anteriormente, la observación del espacio no solo ha tenido importancia en el contexto religioso. Ha servido también para fines arquitectónicos, científicos y de navegación. Nicolás Copérnico, Galileo Galilei y William Herschel son algunos de los personajes más influyentes en el entendimiento de los astros, quienes permitieron el avance del conocimiento del espacio.

Sin embargo, la sed del hombre no se quedó en la observación de las estrellas sino que se aventuró a explorar el espacio ultraterrestre. Inspirados por escritores como Julio Verne, personajes como Robert Goddard y Wernher von Braun Iograron su sueño de enviar objetos y personas al espacio ultraterrestre. Sin embargo, esto jamás hubiese sido posible sin el desarrollo de la tecnología aeronáutica. Si bien hoy en día, cuando se habla de la exploración del espacio aéreo y de la exploración del espacio ultraterrestre, se habla de temas completamente distintos, no se puede negar el origen conjunto que tienen ambas disciplinas. La ambición del hombre de volar, de separarse de la Tierra, ha sido más poderosa que las mismas leyes de la física que gobiernan nuestro planeta.

De esta manera, para poder entender los postulados del derecho espacial -como disciplina del derecho internacional público- es necesario comprender la historia detrás de la exploración del espacio ultraterrestre. Igualmente, es necesario comprender las semejanzas y diferencias físicas y jurídicas que existen entre el espacio aéreo y el espacio sideral. Además, el entendimiento básico de los conceptos científicos que aplican en el espacio ultraterrestre es un requisito indispensable para el estudio de la materia en cuestión.

Por esta razón, se busca realizar un texto que dé una introducción al estudio del derecho espacial. Así, se entiende que se trata de un texto de contenido jurídico. Sin embargo, a lo largo del ensayo se explorarán fuentes científicas, literarias, religiosas e históricas para así poder tener un mejor entendimiento de las aristas que operan en este tema. De esta manera, el presente texto hará una breve explicación de la evolución que ha tenido el hombre del entendimiento de los astros y del espacio, para luego adentrarse en el análisis de la exploración espacial. Se hará énfasis en el periodo transcurrido entre la déca- 
da de los cuarenta y la década de los ochenta, para luego hacer una explicación sumaria de los instrumentos de derecho internacional público que rigen hoy en día. Siguiendo los pasos metodológicos antes descritos, se evidenciará la necesidad imperiosa del derecho espacial, y los grandes logros de esta disciplina.

Así, se observará cómo provenimos del espacio ultraterrestre y cómo ahora nos direccionamos hacia él. De este modo, se pretende defender la tesis de que el derecho espacial ha permitido regular y direccionar la exploración espacial de una manera acertada, teniendo en cuenta los principios del uso pacífico, de no apropiación y de cooperación, aun cuando los mayores avances de la exploración espacial se han dado con fines militares.

\section{LA HISTORIA DEL ESPACIO}

Es innegable que el origen del ser humano radica en el espacio. De hecho, el origen de todas las cosas radica en el espacio, los átomos que componen toda la materia nacieron en un momento determinado que hoy conocemos como el big bang. Sin embargo, el entendimiento del universo no siempre fue el mismo. Diferentes religiones y tradiciones sitúan el origen del mundo en las manos de seres superhumanos, quienes tenían total dominio de todas las cosas. No obstante, la gran mayoría de estas tradiciones lograron identificar su origen en seres celestiales, quienes, desde arriba, construyeron el universo. Los antiguos griegos identifican que la gran mayoría de sus dioses habitaban en un sitio en las nubes, que se llamaba Olimpo. Por otra parte, las culturas latinoamericanas, en especial la cultura muisca, identificaba al Sol y a la Luna, dos cuerpos celestes, como sus dioses. Finalmente, la tradición judeocristiana, en el libro del Génesis, reconoce que Dios es el creador de todo el universo, y que él habita en los cielos. Si bien la concepción de deidades que tenían estas culturas difiere la una de la otra profundamente, todas coinciden en que hay algo más allá del cielo azul. Estas culturas lograron identificar que el origen de la vida provenía de afuera de la Tierra, y que debíamos explorar qué había más allá. De hecho, desde la Antigua Grecia se afirmaba que la Tierra era redonda, y fue Eratóstenes quien logró establecer la circunferencia del globo, mediante la implementación de las latitudes y longitudes (Eratóstenes, circa 195 A. c.). De hecho, el sistema de longitudes y latitudes sigue siendo usado hoy en día, y es de vital importancia para el lanzamiento y seguimiento de satélites.

Otras culturas también se dedicaron a observar los astros y a tratar de graficar lo que veían en el cielo. Los antiguos egipcios fueron la primera cultura, en el año 3.050 A. c., en dibujar una carta astral precisa (Von Spaeth). De hecho, su amplio conocimiento y entendimiento de las estrellas les permitió construir las pirámides de Guiza, replicando el patrón de la constelación del Cinturón de Orión. Por otra parte, los mayas, quienes también construían pirámides, tenían estructuras dedicadas únicamente a la observación de los cielos. Al igual que otras culturas latinoamericanas, como los incas, los mayas 
construían sus edificios para que tuvieran concordancia astrológica con momentos significativos en el año, como los solsticios. Esto también lo hicieron las antiguas civilizaciones que habitaron en las planicies de Salisbury, quienes construyeron Stonehenge (Unesco, 2008). Esta estructura tiene las mismas características de concordancia astronómica que tienen las construcciones en lugares como Ollantaytambo, Machu Picchu en el Perú y Chichén Itzá en México.

Estos hechos indican la fijación que ha tenido el ser humano por entender los astros, y entender lo que sucede en el espacio ultraterrestre. Sin embargo, ha habido ciertos personajes que han permitido el avance significativo del conocimiento de los astros. Uno de los grandes intelectuales que permitió un mejor entendimiento del universo fue Nicolás Copérnico, quien logró demostrar que los planetas no giraban alrededor de la Tierra, sino que todos los planetas giraban alrededor del Sol. Su teoría del heliocentrismo fue duramente criticada y fue muy controversial en el siglo XVI, cuando fue publicada. No obstante, su libro, De revolutionibus orbium coelestium (De las revoluciones de las esferas celestiales), cambió la concepción astronómica de la época, y logró la aceptación de la comunidad científica y religiosa (Crowe, 2001). Otro gran personaje que permitió el avance de la astronomía, y que posibilitó la expansión del conocimiento sobre el espacio, fue el científico italiano Galileo Galilei, quien no solo aportó grandes avances al telescopio, sino que hizo grandes descubrimientos de estrellas y planetas. Galileo, quien se suscribía a la escuela del copernicanismo, creía fir- memente en el heliocentrismo, lo cual le generó grandes problemas con el Vaticano, en donde se sostenía la teoría del geocentrismo. Galileo intentó convencer a las autoridades eclesiásticas de que la Tierra giraba alrededor del Sol, y no como lo sostenían los textos religiosos, los cuales apuntaban a que la Tierra se mantenía quieta y todo giraba alrededor de ella. Los esfuerzos de Galileo no tuvieron muchos resultados, y, de hecho, fue condenado a prisión domiciliaria por hereje. No obstante, Galileo logró observar por primera vez a Neptuno, los anillos de Saturno y cuatro de las lunas de Júpiter. Igualmente, Galileo identificó ciertas manchas sobre el Sol, y fue la primera persona en describir las fases de Venus (Naess, 2005).

Con el pasar de los años, los astros empezaron a cobrar más y más importancia. Con el descubrimiento de América y el establecimiento de colonias británicas, españolas, francesas, portuguesas y holandesas por todo el globo, nació la necesidad de desarrollar un sistema de navegación marítima adecuado. Fue así como los cartógrafos de la época empezaron a dibujar cartas astrales para que los marineros se pudieran guiar en las noches, siguiendo las constelaciones del lugar en el que estuvieran. Las estrellas se miraban por medio de un aparato llamado sextante, que permitía calcular los ángulos entre las estrellas y la Luna. No obstante, estas cartas no eran muy precisas, y muchas veces llevaban a que las naves naufragaran en altamar. Teniendo en cuenta la importancia de la navegación para el Imperio Británico, a mediados del siglo XVII la Corona comisionó a John 
Flamsteed para que diseñara una carta astral más precisa. Flamsteed se embarcó en la tarea, y logró graficar más de tres mil estrellas, así como ser una de las primeras personas en identificar a Urano. El astrónomo británico cambió la navegación por completo, haciendo las cartas astrales quince veces más precisas que las que existían antes. Como consecuencia de sus hallazgos se construyó el observatorio de Greenwich, y se le nombró el primer Astrónomo Real (The History Channel, 2012).

Los avances logrados por Flamsteed fueron tan importantes, que sus cartas astrales fueron utilizadas por los navegantes de los bombarderos británicos que atacaban durante la noche las ciudades alemanas durante la Segunda Guerra Mundial. Estos aviones dependían de las estrellas para poder encontrar su rumbo, y llegar al objetivo establecido. Claro está que en esa época la navegación aérea era muy precaria, por eso, con el advenimiento de la tecnología aeronáutica en 1903, la regulación del espacio aéreo se convirtió en una necesidad imperante. Es así como en 1919, en la ciudad de París, se firmó la Convención para la Reglamentación de la Navegación Aérea Internacional, y, posteriormente, en 1944, se firmó la Convención sobre Aviación Civil Internacional, también conocida como la Convención de Chicago.

Estos instrumentos de derecho internacional tenían como objetivo regular todo lo relativo a la soberanía del espacio aéreo, el paso inocente de aeronaves, la responsabilidad por daños causados en el espacio aéreo, y otros temas concernientes a la disciplina. Estos temas ad- quieren gran importancia para el estudio del derecho espacial, porque, de cierta manera, sirven de precedente jurídico. Además, es necesario establecer que, tradicionalmente, se ha definido el límite del espacio aéreo a una altura de cien kilómetros sobre el nivel del mar, lo que significa que esta distancia es el punto a partir del cual inicia la aplicación del derecho espacial. De igual manera, se debe entender que, en ciertos casos, a un objeto se le puede aplicar tanto el derecho aeronáutico, como el derecho espacial (es el caso del transbordador espacial). Es por esta razón que la concepción de espacio aéreo está fuertemente ligada al estudio del espacio ultraterrestre, y la historia de la exploración de ambas está fuertemente ligada.

Habiendo hecho un breve recuento de la concepción del espacio y la importancia que ha tenido en el desarrollo del ser humano, es importante destacar a personajes como Julio Verne. Él, a través de sus obras, logró inspirar a generaciones futuras para que llevaran a cabo la exploración del espacio ultraterrestre. Con obras como De la Tierra a la Luna, Verne se anticipó casi cien años al alunizaje del hombre, y describió cómo debía ser dicha exploración. Por otra parte, personajes como Robert Goddard y Wernher von Braun fueron fundamentales en el desarroIlo de las tecnologías para los lanzamientos espaciales. Por su parte, Goddard, el inventor del cohete moderno, abrió el camino para que von Braun desarrollara la tecnología del misil V2, el primer objeto creado por el hombre en salir de la Tierra, el cual sirvió, finalmente, de inspiración para los primeros vehículos espaciales. 
Las personas acá descritas son muy pocas en comparación con los cientos de pioneros que lograron aportar al conocimiento que hoy nos permite disfrutar de tecnologías como el GPS, la televisión satelital o la internet. Por esta razón, es necesario entender qué ocurre en el espacio ultraterrestre, cómo llegamos allá, y qué regulaciones aplican en el vacío sideral.

\section{LA CIENCIA DEL ESPACIO}

Como se ha mencionado previamente, para poder entender las regulaciones del espacio, es necesario entender qué es el espacio y qué ocurre allí. De esta manera, se debe tener en cuenta que el espacio es un vacío, un lugar donde no hay absolutamente nada, en donde no se pueden transmitir ondas mecánicas (como el sonido), sino solamente ondas electromagnéticas (como la luz y las ondas de radio). Dentro del espacio se encuentran diferentes tipos de objetos, tales como planetas, estrellas, asteroides, cometas, nebulosas, galaxias y hoyos negros. El conjunto de todos estos objetos es conocido como el universo, cuyo tamaño es desconocido, pero el cual está en constante crecimiento. Incluso, hay científicos que opinan que el universo es infinito. Debe entenderse al espacio ultraterrestre como todo aquello que está más allá de los cien kilómetros sobre el nivel del mar.

El origen del universo, según Georges Lemaitre, ocurre con un suceso conocido como el big bang. Lemaitre afirma que hace billones de años hubo una gran explosión, que causó el origen del universo y su rápida expansión debido a las altas temperaturas generadas durante la misma. Poco a poco se fueron generando los distintos átomos que hoy conocemos, y las partículas se fueron uniendo para crear distintos objetos. Con el pasar del tiempo, los diferentes cuerpos celestes fueron colisionando y creando planetas. El plasma y los gases se fueron concentrando gracias a la gravedad, y se calentaron debido a las reacciones químicas internas, para así formar las estrellas (Woolfson, 2013). No obstante, la certeza de esta teoría no es muy alta, pero es la más aceptada por la comunidad científica.

En el espacio, los planetas y estrellas se agrupan en galaxias, y dentro de las galaxias los planetas se organizan en sistema estelares. La Tierra hace parte de la galaxia conocida como la Vía Láctea, y está dentro del sistema solar. Junto con la Tierra hay otros siete planetas, un cinturón de asteroides y un cuerpo celestial conocido como Plutón (antes considerado planeta), que giran alrededor de una estrella conocida como el Sol. Además, algunos planetas tienen lunas que orbitan alrededor de ellos, como Júpiter, que tiene 67 satélites naturales.

Es importante llamar la atención al hecho de que en el espacio no hay materia, sino que se trata de un vacío. Esto implica un problema muy grande para quienes desean realizar exploraciones, pues en el espacio los vehículos no pueden navegar. Esto se debe al hecho de que para que se pueda navegar se requiere un medio, el cual no existe. Así los vehículos espaciales no navegan sino que se desplazan, impulsando materia (usualmente gases) en dirección opuesta a la 
que el vehículo va. Esto también implica que si un objeto es impulsado en el espacio, este jamás parará de desplazarse hasta que encuentre o ejerza una fuerza equivalente en la dirección opuesta.

En el espacio ultraterrestre ocurren una serie de fenómenos muy curiosos, pero quizá el más común de todos es el de las órbitas. Un objeto que orbita (satélite) alrededor de otro es un objeto cuya velocidad es equivalente a la fuerza de gravedad ejercida por ese objeto. Eso quiere decir que el satélite está en constante caída hacia el objeto respecto del que orbita, pero su velocidad se lo impide, y como tal permanece en constante movimiento. Las órbitas pueden ser circulares o elípticas; si son elípticas, es de comprender que en un punto de la órbita el satélite estará más cerca del objeto orbitado. Este punto es conocido como el perigeo; mientras que el punto más lejano es conocido como apogeo.

Puede haber orbitas alrededor de cualquier objeto que ejerza gravedad, esto quiere decir que hasta nuestra Luna podría tener su propio satélite. De hecho, los módulos lunares que aterrizaron en la Luna durante el proyecto Apolo, en los años sesenta y setenta, lograron entrar en órbita alrededor de la Luna. Sin embargo, es importante resaltar que la velocidad de órbita depende de la cercanía del satélite al objeto orbitado. Esto quiere decir que entre más cerca esté el satélite, más rápido va a girar.

Como tal, en la Tierra es posible que objetos situados en órbita a 37786 kilómetros sobre el nivel del mar, sobre la línea del ecuador, puedan girar a la misma velocidad que el planeta gira sobre su propio eje, y, como tal, dar la impresión de permanecer en un solo lugar. Esta órbita es conocida como la órbita de los satélites geoestacionarios, y fue descubierta y propuesta por el autor de ciencia ficción Arthur C. Clarke, en 1945, antes de la existencia de cualquier satélite hecho por el hombre. Todos estos elementos tratados anteriormente han sido regulados de alguna u otra manera por el derecho espacial. El principio de no apropiación implica que ninguno de los cuerpos celestes podrá convertirse en propiedad de ningún Estado. Por otra parte, la responsabilidad por daños causados por objetos espaciales obliga a los Estados de lanzamiento a seguir las normas de la prudencia y diligencia en el espacio, para así prevenir y evitar daños a terceros.

\section{LA EXPLORACIÓN DEL ESPACIO}

Uno de los grandes sueños del ser humano ha sido poder volar y explorar los cielos; esto se ve reflejado en la mitología griega, en el mito de Ícaro. Este sueño se cumplió en 1903 cuando los hermanos Wright lograron el primer vuelo de un aeroplano. Sin embargo, el sueño de ir a las estrellas, visitar la Luna, y explorar el espacio, no se cumplió hasta la mitad del siglo XX. No obstante, varios autores exploraron la idea de las aventuras espaciales. Uno de los textos de ciencia ficción más importantes sobre la materia, De la Tierra a la Luna, fue escrito por Julio Verne en el año de 1865. En él cuenta el relato de un grupo de hombres pertenecientes al Gun-Club, quienes, después de la Guerra Civil 
de los Estados Unidos, deciden realizar un viaje a la Luna en un proyectil disparado por el gigantesco cañón Columbiad. Durante la novela, Verne describe todos los preparativos que deben surtir el capitán Nicholl, Impey Barbicane y Miguel Ardan, los primeros astronautas de la literatura (Verne, 1865). A lo largo del texto, se observa cómo los tres personajes hacen todos los cálculos para poder realizar su viaje. Desde la cantidad de pólvora necesaria para impulsar el proyectil a la Luna, pasando por el material del cañón, hasta el punto en el cual debería ser ubicado el Columbiad para alcanzar su objetivo de la mejor manera. Luego de enfrentar los diferentes contratiempos que conlleva un viaje a la Luna, los tres protagonistas son disparados hacia el espacio. No obstante, el proyectil no logra impactar en su objetivo sino que entra en una órbita alrededor del cuerpo celeste (Verne, 1865). Verne termina su novela ahí, para luego hacer una continuación de la historia en 1870 , en Alrededor de la Luna.

En este segundo texto, Verne relata todos los detalles del trayecto de los primeros astronautas hacia la Luna, y su vida orbitando el gran satélite. Luego de llegar a la conclusión de que en un lado de la Luna no hay vida, los tres personajes se concentran en explorar el lado oscuro de este cuerpo celeste. Allí concluyen que alguna vez hubo civilizaciones que habitaron esta gran roca pero que fueron destruidas con el paso del tiempo. Luego de hacer sus observaciones, Verne prosigue a narrar cómo los protagonistas usan los cohetes que aún quedaban en el proyectil para salirse de la órbita de la Luna, y luego entrar a la Tierra para caer en el océano y esperar a ser rescatados (Verne, 1870).

Si bien gran parte de las obras de Verne se adecúan al género de la ciencia ficción, parece casi increíble cómo él, casi cien años antes del primer alunizaje, logra describir con tanta precisión ciertos detalles que se repiten en la vida real. El hecho de poder predecir las necesidades de viajar en una cápsula propulsada desde la Tierra, que pudiera orbitar alrededor de la Luna, y que luego usara cohetes para escapar de la gravedad lunar, para caer a la Tierra en el océano, es impresionante. Pareciera que los ingenieros de las agencias espaciales estadounidenses y soviéticas hubiesen calcado las descripciones de Verne y las hubieran hecho realidad.

No obstante, como se mencionó anteriormente, tuvieron que pasar casi cien años y cientos de avances tecnológicos para que el hombre pudiera tan siquiera poner un objeto en el espacio ultraterrestre. El primero de estos avances, y quizás el más importante, fue aquel logrado por Robert Goddard, el inventor del cohete de combustible líquido. Si bien él no fue el inventor de los cohetes, que de hecho existían desde la antigua China, y eran propulsados por pólvora y otros combustibles sólidos, Goddard logró utilizar el oxígeno líquido para propulsar sus misiles. Los experimentos de Goddard arrojaron resultados nunca antes vistos; las altitudes a las que llegaban sus cohetes nunca habían sido exploradas, pues los combustibles sólidos solo podían generar una cierta cantidad de propulsión (Huntley, 1995). 
El científico estadounidense logró hacer su primer lanzamiento exitoso el 16 de marzo de 1926, hace casi noventa años (Goddard, 1914). En este primer lanzamiento, el cohete alcanzó una altitud de 12,5 metros. Sin embargo, Goddard no estuvo satisfecho con este resultado, y continuó con sus experimentos y desarrollos, para luego, en 1937, lograr impulsar uno de sus cohetes a casi 2700 metros de altitud (Huntley, 1995). Eso es como si lanzaran un cohete desde el nivel del mar, y llegase a la altura de Bogotá en tan solo unos segundos.

Como se mencionó anteriormente, el trabajo de Goddard fue fundamental para la exploración del espacio, pues para esa época no existía la tecnología para impulsar un objeto a más de doscientos metros. No obstante, el genio de Goddard no paró con la invención del cohete de combustible líquido, Goddard también patentó el cohete multietapa, herramienta fundamental que hoy sigue siendo utilizada por todas las agencias espaciales del mundo (Huntley, 1995). Debe recalcarse que los cohetes de Goddard no solo volaban alto y rápido, sino que eran precisos y estables, gracias a los giroscopios y demás sistemas que este científico instalaba dentro de sus misiles (Huntley, 1995). Es evidente que Goddard puede ser considerado uno de los padres de la exploración espacial, y de la ciencia de los misiles como tal. Los diseños de Goddard fueron utilizados por alemanes, estadounidenses y rusos para la construcción de sus cohetes, y, desafortunadamente, también para el diseño de las diferentes armas utilizadas por estos países.
Con el pasar de los años, diferentes científicos fueron perfeccionando la tecnología de misiles y cohetes. Su velocidad, precisión y altura fue incrementando, al igual que el interés de los diferentes ejércitos al ver su gran potencial destructivo. Así, para la Segunda Guerra Mundial, los diferentes ejércitos estaban utilizando cohetes como armas de artillería en contra de sus enemigos.

Los soviéticos tenían los Katyusha (o acordeones de Stalin, como fueron bautizados por los alemanes debido al ruido que producían), los alemanes utilizaban los Nebelwerfer, y, los aliados, los Calliope. Todas estas armas tenían un inmenso poder destructivo, pero ninguno como el misil alemán V2 (Lüdeke, 2007). Esta arma fue diseñada por el científico alemán Werhner von Braun, para atacar las diferentes ciudades del Reino Unido (Lüdeke, 2007). El V2 fue la continuación del V1, un misil propulsado por un motor jet, que era lanzado indiscriminadamente contra las ciudades británicas. Sin embargo, el V1 representaba una serie de problemas para el régimen nazi, pues su velocidad le permitía a la Real Fuerza Aérea derribar estas armas (Cartier, 1965). Por esta razón, Hitler le encomendó a Von Braun el diseño de una "arma de venganza" que pudiera ser lanzada desde cualquier parte para que impactara en Londres, sin que la Real Fuerza Aérea pudiera reaccionar.

El desarrollo del V2 comenzó en 1937, como parte de los proyectos de investigación de Von Braun. Sin embargo, con el advenimiento de la Segunda Guerra Mundial, y al Von Braun ser miembro del partido Nazi, se inició la conversión 
de este cohete en un arma. El Gobierno alemán mostró poco interés en el proyecto, pero finalmente decidió financiarlo y estableció los laboratorios de investigación y la fábrica de estos cohetes en la región de Peenemünde. Poco a poco fueron progresando los avances en este cohete, el cual estuvo listo en 1943.

Hitler asistió al lanzamiento de uno de estos misiles, y se percató de las ventajas tácticas que un arma como estas le daba; inmediatamente ordenó la puesta en producción masiva (Cartier, 1965). Los V2 eran fabricados por prisioneros de campos de concentración en complejos subterráneos para evitar el bombardeo de los aliados. Finalmente, en septiembre de 1944, el primer V2 fue lanzado contra Londres, ocasionando graves daños y un alto número de muertos y heridos (Lüdeke, 2007).

El V2 fue un aparato revolucionario para la época. Era un misil de una sola etapa propulsado por oxígeno líquido (inventado por Goddard), que alcanzaba velocidades de casi seis mil kilómetros por hora. Podía ser lanzado desde cualquier parte, gracias a su plataforma de lanzamiento móvil, y era extremadamente preciso, lo que ocasionaba grandes daños a los británicos (Lüdeke, 2007). Este misil es considerado como el primer misil balístico que existió, lo que significaba que el cohete era disparado hacia el espacio, donde hacía una parábola para luego caer sobre su objetivo. De hecho, el v2 fue el primer objeto hecho por el hombre en salir de la Tierra (Lüdeke, 2007). Por esta razón, esta arma se convirtió en una de las últimas líneas de defensa de Hitler contra los aliados, quienes, para principios de 1945, ya estaban pisando territorio alemán.

Con el fin de la guerra, los aliados compitieron entre ellos por apoderarse de toda la tecnología que poseían los alemanes. Pero probablemente el aparato más preciado por capturar era el misil V2, y la tecnología que contenía. Wernher von Braun sabía el botín que este representaba para los aliados, y, por esa razón, decidió escabullirse para luego entregarse a un grupo de soldados estadunidenses (Lüdeke, 2007).

Luego de la Segunda Guerra Mundial, se puede hablar de que inició un periodo conocido como la guerra fría. Este se caracterizó por una serie de sucesos y conflictos, pero quizás el más interesante fue aquel conocido como la carrera espacial. Después de que los estadunidenses y soviéticos capturaron los diseños y el conocimiento de los alemanes, ambos países empezaron a desarrollar sus propios cohetes. Sin embargo, el reflejo del progreso de los soviéticos se vio el 4 de octubre de 1957, cuando lanzaron y pusieron en órbita el primer satélite hecho por el hombre, Sputnik 1. Este satélite giraba alrededor de la Tierra, transmitiendo ondas de radio que podían ser recogidas por cualquier persona (Siddiqi, 2003).

El lanzamiento de este objeto desencadenó una crisis dentro de los Estados Unidos, y marcó el principio de la "era espacial". Sin embargo, para agravar esta situación, un mes después del lanzamiento de Sputnik 1 la Unión Soviética puso en el espacio a Sputnik 2. A bordo de este vehículo se encontraba el primer ser viviente en 
el espacio, la perra Laika. Como respuesta, los Estados Unidos lanzaron, en enero de 1958, el Explorer 1, satélite que orbitaría la Tierra, y que transmitiría hasta que se agotaran sus baterías. Sin embargo, esta era la segunda vez que se trataba de hacer el lanzamiento, puesto que los Estados Unidos ya habían tenido un intento de lanzamiento fallido en diciembre de 1957. De igual forma, y a diferencia de Sputnik, el Explorer transmitía datos que percibía en el espacio, y no solo una frecuencia de radio.

Continuando con esta carrera espacial, los Estados Unidos crearon la Agencia Nacional de Aeronáutica y del Espacio (NASA, por sus siglas en inglés). Esta se encargó de todo el desarrollo de las misiones al espacio. No obstante, en su creación, los Estados Unidos fueron derrotados nuevamente por los soviéticos, cuando en abril de 1961 lanzaron a Yuri Gagarin al espacio. El cosmonauta soviético orbitó la Tierra, para luego aterrizar sano y salvo, hecho que fue respondido por los estadunidenses al poner en el espacio a Alan Shepard un mes después del vuelo de Gagarin (Siddiqi, 2003).

Los avances de ambos bandos llevaron al lanzamiento de satélites más avanzados que los anteriores, y permitiéndole a sus cosmonautas y astronautas realizar nuevas y más tareas en el espacio. Esto llevo a que, en un famoso discurso, el presidente de los Estados Unidos, John F. Kennedy, prometiera poner un hombre en la Luna antes de que se acabara la década de los sesenta. Así fue como, por intermedio de los programas de la NASA, Mercury, Gemini y, finalmente, Apolo, el 20 de julio de 1969 el hombre cami- nó en la Luna. Fue el vehículo denominado Apolo 11 el que transportó a Neil Armstrong, Buzz Aldrin y Michael Collins, y el que permitió que se "diera un pequeño paso para el hombre pero un gran paso para la humanidad"3 (Brzezinski, 2007). Apolo 11 fue propulsado por un cohete multietapa (como los que inventó Goddard), Saturn V, desarrollado por Wernher von Braun. En ese viaje, que cumplía con los presagios de Julio Verne, dos de los tripulantes lograron caminar sobre la superficie lunar, y recolectar una serie de materiales espaciales que hasta el momento eran desconocidos para el hombre.

La carrera espacial culminó en 1975 cuando una nave estadounidense y una nave soviética se unieron en el espacio y convivieron durante unos días. El proyecto Apollo-Soyuz marcó la historia de la exploración espacial (Brzezinski, 2007), y fue uno de los primeros esfuerzos visibles por hacer efectivo el principio de cooperación enmarcado en el Tratado de 1967.

A pesar de que la carrera espacial era un esfuerzo de las dos grandes potencias para llegar al espacio y tener logros significativos en él, no se puede desconocer cuál es la verdadera razón de la existencia de la misma. Si bien soviéticos y estadounidenses pretendían avanzar científicamente y probar que estaban más adelantados que el otro, la carrera espacial fue un reflejo hipócrita de la carrera armamentista que los dos países llevaron durante la década de

3 Palabras pronunciadas por Neil Armstrong al dar sus primeros pasos sobre la superficie de la Luna, el 20 de julio de 1969. 
los cincuenta y sesenta. Los cohetes que eran utilizados para lanzar a los satélites y vehículos espaciales, eran el reflejo de la capacidad nuclear de cada Estado. Los satélites demostraban la habilidad que tenían para desarrollar misiones de espionaje sobre el otro. Pero, como agravante, los vuelos tripulados le demostraban a la contraparte que el Estado lanzador tenía la capacidad para desplegar tropas en cualquier parte del planeta, inclusive en la Luna.

La verdad es que la carrera espacial fue alimentada por el miedo de los dos países. Si bien la guerra fría se luchó entre las grandes potencias, en muchas partes del mundo, en ninguna fue tan directa como en el espacio. Prueba de que esto es así es el proyecto Apollo-Soyuz, que en 1975 logró unir una nave estadounidense y una nave soviética en el espacio, mientras que en la Tierra se entraba en un proceso détente. Por unos años la exploración espacial perdió protagonismo, aun cuando los proyectos Skylab y Soyuz continuaban.

Sin embargo, con el fracaso de la détente en la década de los ochenta, el espacio se volvió a convertir en un campo de batalla. El presidente Reagan propuso la Strategic Defense Initiative, en la que pretendía poner satélites en el espacio, capaces de destruir misiles soviéticos, y, dado el caso, atacar territorios de la URSS. Este proyecto fue claramente desechado, pues contrariaba todos los principios establecidos por el Tratado de 1967. Esta iniciativa fue tan duramente criticada, que recibió el apodo de "Star Wars", caricaturizando las propuestas del Gobierno de los Estados Unidos. Igualmente, en esa misma década se realizó el primer lanzamiento del transbordador espacial.

Este nuevo vehículo fue lanzado por primera vez en abril de 1981, y tenía como objetivo servir de vehículo reutilizable para desarrollar las diferentes misiones de la NASA. El transbordador espacial tenía la capacidad de ir al espacio ultraterrestre como un cohete, desplazarse en el espacio como un vehículo espacial, para luego regresar a la Tierra y comportarse como una aeronave. Este vehículo fue quizá el mayor avance de la NASA desde Apolo 11, pues logró reducir los costos de operación y permitió que más astronautas viajaran al espacio para lanzar, reparar y retirar satélites de la órbita. Se construyeron cinco transbordadores operacionales, sin embargo, dos de ellos fueron destruidos en accidentes durante misiones. El primero fue el transbordador Challenger, que en 1986 explotó unos segundos luego de decolar (NASA); el segundo fue el transbordador Columbia, que en 2003 se desintegró durante el reingreso a la Tierra, debido a que había perdido una baldosa térmica durante el lanzamiento (NASA). La última misión de un transbordador espacial la realizó el transbordador Atlantis, en julio de 2011. Es importante anotar que en los ochenta los soviéticos lanzaron su propio transbordador espacial; este solo realizó una misión sin tripulación, y fue denominado Buran. Este vehículo era una copia casi exacta del transbordador espacial de la NASA, y el proyecto fue desechado luego de la caída de la Unión Soviética (Brzezinski, 2007).

Hoy en día la exploración del espacio ha cambiado radicalmente, la mayoría se hace por vehícu- 
Ios no tripulados que son controlados desde la Tierra. La mayoría de los lanzamientos son para poner en órbita distintos satélites, o para enviar y traer tripulantes de la Estación Espacial Internacional. El avance en la tecnología y el principio de cooperación han permitido que otros países, diferentes a Rusia y a los Estados Unidos, puedan tener programas espaciales. Igualmente, ya comienza a deslumbrarse un nuevo futuro en la exploración espacial: el turismo espacial. Han sido pocas las personas que han pagado millonarias sumas de dinero para ser llevados al espacio, pero con proyectos como los propuestos por compañías como Virgin Galactic, las oportunidades de ir al espacio cada día se vuelven más grandes (Virgin Galactic, 2013). Estos nuevos proyectos van a cambiar nuestra comprensión del espacio, y de la exploración espacial. Seguramente, estos proyectos cambiarán el régimen jurídico que opera más allá de los cien kilómetros sobre el nivel del mar.

\section{LA REGULACIÓN DEL ESPACIO}

El derecho es la ciencia de la regulación del comportamiento humano para facilitar la convivencia entre los hombres. Este enunciado da pie para explicar que donde sea que esté el hombre, allá estará el derecho. Al ir al espacio y desarrollar diferentes actividades por fuera de la Tierra, el hombre no solo ha desarrollado cohetes y satélites, sino que diseñó un sistema jurídico aplicable a todas las personas y objetos por fuera de los cien kilómetros sobre el nivel del mar. Resulta paradójico regular con leyes humanas, algo que se sale de lo tradicio- nalmente considerado como humano: la Tierra. Sin embargo, se observará cómo la regulación espacial ha permitido el desarrollo pacífico de la exploración espacial.

Los orígenes del derecho espacial se remontan a las convenciones de París y Chicago, anteriormente descritas, las cuales regularon el tráfico aéreo y la responsabilidad causada en el espacio aéreo y por aeronaves. Igualmente, el derecho espacial puede remontarse al derecho marítimo, pues se comparten ciertas regulaciones como las del paso inocente, el salvamento de personas y la no apropiación de las aguas internacionales. El derecho espacial es una disciplina perteneciente al derecho internacional público que, como ya se ha explicado en varias ocasiones, busca regular las actividades del hombre en el espacio ultraterrestre.

Los instrumentos de derecho espacial, considerados como disciplina independiente, pueden trazar sus orígenes a la Carta de las Naciones Unidas y a distintas resoluciones de la Asamblea General de las Naciones Unidas. No se habla de regulaciones en otros organismos internacionales, pues la posibilidad de ir al espacio no existía antes de la década de los cincuenta. Sin embargo, con la puesta en órbita del satélite Sputnik 1 por parte de la URSS, la comunidad internacional se vio en la imperiosa necesidad de establecer un régimen jurídico que limitara las acciones abusivas de las potencias, y les garantizara el acceso al espacio a los países en vía de desarrollo.

Así, en 1963 fueron aprobadas dos resoluciones en la XVIII sesión de la Asamblea General de 
las Naciones Unidas, que establecían los principios jurídicos que debían regir en el espacio ultraterrestre, y la prohibición del uso de armas de cualquier caso en el espacio (Resoluciones 1962 y 1883, respectivamente). Posterior a esto, en 1966, en su XXI sesión, la Asamblea General aprobó la Resolución 2222, por medio de la cual se aprobó el Tratado sobre los principios que deben regir las actividades de los Estados en la exploración y utilización del espacio ultraterrestre, incluso la Luna y otros cuerpos celestes (también conocido como el Tratado del Espacio de 1967, o Tratado de 1967). No obstante, el hecho de que las resoluciones de la Asamblea General sean parte del soft law del derecho internacional, y como tal, de que no tengan efecto vinculante para los Estados, hizo que el documento fuese puesto para la firma de la comunidad internacional. Finalmente, el tratado entró en vigencia en enero de 1967, como instrumento y fuente de derecho internacional.

El Tratado de 1967, también denominado como la "constitución del espacio", introdujo una serie de principios que, hoy por hoy, rigen la actividad espacial. Estos son: el principio de no apropiación, uso pacífico del espacio ultraterrestre y cooperación internacional. El principio de no apropiación indica que ningún Estado, bajo ninguna circunstancia, podrá apropiarse de ninguna parte del espacio, ninguna órbita, ningún planeta, ni cualquier otro cuerpo celeste. Este elemento llegó a buen momento, pues la entrada en vigencia del tratado, dos años antes del primer alunizaje, previno que los Estados Unidos se apropiaran de Luna y la colonizaran como territorio propio.
De haberse incluido este principio en el tratado, no se sabe cómo hubiese terminado la carrera espacial, con una posible confrontación bélica en el espacio entre la URSS y los Estados Unidos por el control del único satélite natural que tiene la Tierra. Igualmente, este principio previene que Estados declaren la soberanía sobre lugares en el espacio ultraterrestre, a través de mecanismos constitucionales o legales, como Io hizo Colombia con la Constitución de 1991. Sobra decir que esa norma constitucional que cobra soberanía sobre "su órbita geoestacionaria" [sic], no tiene aplicación pues no se atiene a las normas del derecho internacional, como el mismo texto lo ordena.

El segundo principio es el del uso pacífico del espacio ultraterrestre, que implica que ningún Estado podrá tener armas, ni llevar armas al espacio ultraterrestre. Si bien este instrumento ha prevenido que en el espacio se ponga armamento, como en el caso de la Strategic Defense Initiative del presidente Reagan, el principio no ha sido cumplido a cabalidad. En primera instancia, el espionaje por medio de satélites es un hecho innegable, y si bien no constituye una acción bélica, el derecho internacional humanitario considera las acciones de inteligencia como acciones de guerra. Igualmente, el establecer que ningún Estado podrá posicionar ningún tipo de armas en el espacio implica que los misiles balísticos están prohibidos por este tratado. Esto se debe a que ese tipo de armas lanza un cohete hacia el espacio, en donde se desprende una ojiva que luego reentrará a la Tierra, para impactar su objetivo. No obstante, todas las na- 
ciones militarmente desarrolladas tienen este tipo de armamento, y están más que dispuestas a utilizarlas en caso de ser necesarias. No obstante, no se puede ignorar el hecho de que, gracias al principio de uso pacífico, se ha logrado mantener la paz y la estabilidad en el espacio, a pesar de los conflictos entre los diferentes Estados en la Tierra.

De igual manera, el Tratado de 1967 incluye el principio de cooperación, el cual incita a las partes contratantes a prestar ayuda y apoyo para el desarrollo del conocimiento del espacio y la exploración del mismo. Este principio se ha visto visualizado en varios casos, como, por ejemplo, en el Proyecto Apollo-Soyuz (Brzezinski, 2007), la Estación Espacial MIR, y, actualmente, en la Estación Espacial Internacional. Igualmente, países desarrollados han colaborado con países en vía de desarrollo para que accedan al espacio por medio del lanzamiento de satélites, como es el caso de China y Rusia, colaborando con Venezuela para el lanzamiento del satélite Simón Bolívar. Este principio radica en el hecho de que el espacio y todos sus cuerpos celestes son patrimonio de toda la humanidad, y por lo tanto todos deben cooperar para lograr su aprovechamiento y conocimiento. Igualmente, el principio de cooperación implica que los Estados tienen la obligación de prestar la debida asistencia a cualquier astronauta que se encuentre en necesidad, por haber sido puesto en peligro debido a la naturaleza de su ocupación. De nuevo, esto se une al hecho de que los astronautas son considerados enviados de la humanidad, y no del Estado de lanzamiento al que pertenezcan.
Asimismo, los Estados contratantes se ven obligados a restituir cualquier objeto espacial que caiga sobre su territorio al Estado de lanzamiento al que pertenezca el objeto.

Este último principio dio vía para que se firmara el segundo tratado del espacio, el Acuerdo sobre el salvamento y la devolución de astronautas y la restitución de objetos lanzados al espacio ultraterrestre, del año de 1968. Por medio de este instrumento se desarrolló el principio de cooperación, y se establecieron las pautas para establecer que un astronauta es un enviado de la humanidad. Por supuesto, en ese tiempo no se vislumbraba la posibilidad de que hubiese turistas espaciales. Sin embargo, hoy en día a ellos les son aplicables los derechos y prerrogativas enmarcados en este documento.

Las nuevas tecnologías permitieron que mayores cantidades de satélites y vehículos fueran lanzados al espacio. Sin embargo, esta situación implicó que existía la posibilidad de que estos objetos sufrieran daños en el espacio, y que, inclusive, causaran daños a otros objetos. Este hecho se vio materializado por los sucesos del Apolo 13, en el que los tres astronautas que piloteaban el vehículo casi mueren a causa de un daño. Así fue como en 1972 entró en vigor el Convenio sobre la responsabilidad internacional por daños causados por objetos espaciales, estableciendo responsabilidad objetiva de los Estados de lanzamiento por daños causados por objetos espaciales en la Tierra. Sin embargo, el régimen de responsabilidad en el espacio es uno de corte subjetivo, muy similar al que existe en los países de derecho continental. Este tra- 
tado tiene amplia concordancia con el Convenio sobre el registro de objetos lanzados al espacio ultraterrestre, el cual obliga a los distintos Estados de lanzamiento (término definido por el Tratado de 1967) llevar un registro claro de todos sus objetos lanzados.

Igualmente, las normas de la Unión Internacional de Telecomunicaciones influyen en el derecho espacial. Es así como se ha visto un avance en la protección de la órbita de los satélites geoestacionarios por parte de este organismo internacional. Si bien la Conferencia de Montreaux no arrojó ninguna conclusión frente a esta órbita especial, la Conferencia de MálagaTorremolinos concluyó que es un recurso natural limitado que debe ser usado de manera eficiente para el beneficio de la humanidad. No obstante, la Conferencia de 1982 concluyó que la órbita debe ser utilizada de manera eficiente, teniendo en cuenta las necesidades especiales de los países en vía de desarrollo, y la situación geográfica de los países.

No obstante, todavía falta regulación frente a la misma. Algo que los países ecuatoriales, con excepción de Brasil, llevan solicitando desde 1976. Es un hecho que los países ecuatoriales se encuentran en una posición especial, que hace que los satélites ubicados en la órbita de los satélites geoestacionarios permanezcan permanentemente sobre su territorio, si es que han sido situados en esa plaza. Por lo tanto, se requiere una mayor legislación al respecto que permita solucionar efectiva y eficientemente los conflictos entre los Estados.
Por otra parte, es importante llamar la atención sobre el hecho de que el ente encargado de regular todo lo concerniente al espacio ultraterrestre en el derecho internacional sea la Oficina de Asuntos del Espacio Ultraterrestre de las Naciones Unidas. Esta oficina está compuesta por la Comisión sobre la Utilización del Espacio Ultraterrestre para Fines Pacíficos, dividida en dos subcomisiones, una técnica y otra jurídica, que se encargan de tratar todos los temas concernientes al espacio ultraterrestre, el lanzamiento de objetos y cualquier otra regulación relacionada con el tema.

\section{CONCLUSIONES}

Habiendo hecho este breve estudio del derecho espacial y la historia de la exploración espacial, se puede llegar a la firme conclusión de que el ser humano no puede separarse del espacio. Sin embargo, han sido circunstancias de religión, comercio, ciencia, guerra, y simple curiosidad, las que han llevado al hombre a ahondar en su conocimiento de los astros.

Como se sostenía en un principio, el hombre proviene de las estrellas y ahora busca dirigirse hacia ellas, como el rumbo a seguir de la humanidad. El espacio le ha permitido al ser humano descubrir su propio planeta, entender de una forma u otra su propia existencia, y llegar a lugares nunca imaginados. No obstante, la mayoría de los avances logrados en el siglo XX fueron consecuencia de la guerra, de la violencia. El espacio se convirtió en un campo de batalla donde unos trataban de sobrepasar al 
otro a cualquier costo. Sin embargo, fue gracias

a la intervención de la ley, a través del derecho espacial, que se logró poner fin a esto.

Aun así, es triste observar cómo los grandes avances en la exploración espacial se han dado durante tiempos de alta tensión, como lo fueron las décadas de los cincuenta, sesenta y ochenta. No obstante, hoy en día se están convirtiendo tecnologías militares al uso civil. Satélites de GPS que antes eran utilizados para guiar bombas, ahora les permiten a exploradores llegar a lugares remotos en la geografía del planeta. Ahora, los nuevos horizontes presentan oportunidades para continuar explorando el sistema solar, y, por supuesto, esperar a que en los próximos años se logre poner un hombre en Marte.

Por otra parte, se debe tornar la mirada hacia el turismo espacial, y llamar la atención de las autoridades en el derecho espacial para que regulen la materia. Es así como se prevendrán tragedias, y se continuará con el gran éxito que ha tenido esa rama de las ciencias jurídicas, al prevenir conflictos y reparar exitosamente los daños causados. A manera de conclusión, se afirma que el éxito de la exploración espacial se ha dado gracias a la presencia del derecho espacial, y a la sed del hombre por conocer más e ir más allá, aun cuando las circunstancias coyunturales han anunciado conflicto. Se dice que el hombre avanza como especie cada vez que hay una guerra, pero la verdad el desarrollo en el espacio ha demostrado que eso no necesariamente es así.

\section{Referencias}

Brzezinski, M. (2007). Red Moon Rising: Sputnik and the Hidden Rivalries that Ignited the Space Race. New York: Times Books.

Cartier, R. (1965). The Second World War 19431945. Paris.

Crowe, M. J. (2001). Theories of the World from Antiquity to Copernican Revolution. New York: Dover Publications.

Eratóstenes (circa 195 A. c.). Geografía de Eratóstenes. Princeton University.

Goddard, R. H. (1914). Patente $n .^{\circ}$ 1, 102, 653. Estados Unidos.

Huntley, J. (1995). The Enigma of Robert H. Goddard. En J. Huntley. Technology and Culture.

Lüdeke, A. (2007). Weapons of World War II. London.

Naess, A. (2005). Galileo Galilei. When the World Stood Still. New York: Springer Berlin Heidelberg.

Siddiqi, A. A. (2003). Sputnik and the Soviet Space Challenge. Gainesville: The University of Florida Press.

- (2003). The Soviet Space Race with Apollo. Gainsville: The University of Florida Press.

The History Channel (Dirección) (2012). Mankind. The History of all of us [Película]. 
UNESCO (2008). Stonehenge World Heritage Site Management Plan.

Verne, J. (2010). From The Earth to The Moon and A Trip Around It. Fairford: The Echo Library.

Virgin Galactic (2013). Virgin Galactic - Overview. Recuperado de http://www.virgingalactic.com/overview/
Von Spaeth, O. Recuperado de http://www. moses-egypt.net/star-map/senmut1-mapdate_en.asp

Woolfson, M. (2013). Time, Space, Stars and Man: The Story of Big Bang (2. ${ }^{a}$ ed.). World Scientific Publishing. 